\title{
Generalized Hölder's inequality in Orlicz sequence spaces
}

\author{
Siti Fatimah ${ }^{1}$, Al Azhary Masta ${ }^{2}$ Ifronika $^{3}$, Risnina Wafiqoh ${ }^{4}$, and Putri Cahyani Agustine ${ }^{5}$ \\ \{Sitifatimah@upi.edu1, alazhari.masta@upi.edu²,nina.wafiqoh492@gmail.com³ \\ putri.cahyaniagustine@stkipmbb.ac.id $\left.{ }^{5}\right\}$
}

Faculty of Mathematics and Science Education, Universitas Pendidikan Indonesia, J1. Dr. Setiabudi 229, Bandung 40154, INDONESIA ${ }^{1,2}$,

Analysis and Geometry Research Group, Bandung Institute of Technology, Bandung, INDONESIA ${ }^{3}$, Department of Mathematics Education, STKIP Muhammadiyah, Jl. KH. Ahmad Dahlan 51, Kabupaten Bangka Tengah, Bangka Belitung, 33684, INDONESIA ${ }^{4,5}$

\begin{abstract}
Orlicz spaces is one of Banach space were firstly initiated by Birnbaum and W. Orlicz. There are two categories of Orlicz spaces, i.e. continuous Orlicz spaces and Orlicz sequence spaces. Many authors have discussed about continuous Orlicz spaces, especially Hölder's inequality in these spaces. In this paper, we present a sufficient condition for generalized Hölder's inequality in Orlicz sequence spaces. One of the keys to finding our results is to use the norm of the characteristic sequence of the balls in $\mathbb{Z}$.
\end{abstract}

Keywords: Hölder's Inequality, Sufficient Condition, Orlicz Sequence Spaces

\section{Introduction}

Orlicz spaces is one of Banach space were firstly initiated by Birnbaum and W. Orlicz in [1]. We can consider Orlicz spaces as a generalizations of Lebesgue spaces. There are two types of Orlicz spaces which are 'continuous' Orlicz spaces denoted by $L_{\Phi}$ and sequence Orlicz spaces denoted by $\ell_{\Phi}$.

We called a function $\Phi:[0, \infty) \rightarrow[0, \infty)$ as a Young function if $\Phi$ is a convex, leftcontinuous, $\lim _{t \rightarrow 0} \Phi(t)=0=\Phi(0)$, and $\lim _{t \rightarrow \infty} \Phi(t)=\infty$. Let $\Phi$ be a Young function, we define $\Phi^{-1}(r):=\inf \{s \geq 0: \Phi(s)>r\}$ for every $r \geq 0$.

Let us firstly recall the definition of Orlicz spaces and Orlicz sequence spaces. For $\Phi$ is a Young function, continuous Orlicz space $L_{\Phi}\left(\mathbb{R}^{n}\right)$ is a set of all measurable functions $f: \mathbb{R}^{n} \rightarrow$ $\mathbb{R}$ such that

is finite.

$$
\|f\|_{L_{\Phi}\left(\mathbb{R}^{n}\right)}:=\inf \left\{b>0: \int_{\mathbb{R}^{n}} \Phi\left(\frac{|f(x)|}{b}\right) d x \leq 1\right\}
$$

Now, let $\Phi$ be a Young function. The Orlicz sequence spaces $\ell_{\Phi}(\mathbb{Z})$ is the set of all sequences $x:=\left(x_{n}\right)_{n \in \mathbb{Z}}$ such that

$$
\|x\|_{\ell_{\Phi}(\mathbb{Z})}=\inf \left\{b>0: \sum_{k=1}^{\infty} \Phi\left(\frac{\left|x_{k}\right|}{b}\right) \leq 1\right\}<\infty .
$$


If we take $\Phi(t)=t^{p}$ for every $t \geq 0$ and $1 \leq p<\infty$ then we have $\ell_{\Phi}(\mathbb{Z})=\ell_{p}(\mathbb{Z})$ is $p$ summable sequence spaces. Furthermore, $\ell_{\Phi}(\mathbb{Z})$ is Banach spaces.

Some expert have discussed about Orlicz spaces (see [2-7], etc.). They have revealed sufficient and essential condition. One of them, Ifronika et al. [8] propose to generalized Hölder's inequality in Orlicz spaces. In addition, Masta et al. suggest to generalized Hölder's in $p$-summable sequence spaces. There for, in this paper will be discussed about the generalized Hölder's inequality in sequence Orlicz spaces and in weak sequence Orlicz spaces.

In the process of finding result, we involve the Young function, Luxemburg norm in sequence Orlicz spaces, and some lemmas as follows.

Lemma 1. [9] Let $\Phi$ be a Young function and $\Phi^{-1}(s):=\inf \{r \geq 0: \Phi(r)>s\}$, then we have

1. $\Phi^{-1}(0)=0$.

2. $\Phi^{-1}\left(s_{1}\right) \leq \Phi^{-1}\left(s_{2}\right)$ for $s_{1} \leq s_{2}$.

3. $\Phi\left(\Phi^{-1}(s)\right) \leq s \leq \Phi^{-1}(\Phi(s))$ for $0 \leq s<\infty$.

Lemma 2. If $x:=\left(x_{k}\right) \in \ell_{\Phi}(\mathbb{Z})$ and $\|x\|_{\ell_{\Phi}(\mathbb{Z})} \neq 0$, then $\sum_{k=1}^{\infty} \Phi\left(\frac{\left|x_{k}\right|}{\|x\|_{\ell_{\Phi}(\mathbb{Z})}}\right) \leq 1$.

\section{Results and Discussions}

Now, we have already known the properties of continuous Orlicz spaces. For getting the result, we will apply these properties to Orlicz sequence spaces. First, we present several lemmas in the following. We will also prove that the mapping on Orlicz sequence spaces define a norm on $\ell_{\Phi}(\mathbb{Z})$.

Theorem 3. Let $\Phi_{1}, \Phi_{2}$ and $\Phi_{3}$ be Young functions and satisfy the condition $\Phi_{1}^{-1}(t) \Phi_{2}^{-1}(t) \leq \Phi_{3}^{-1}(t)$ for every $\mathrm{t} \geq 0$. If $X:=\left(x_{n}\right) \in \ell_{\Phi_{1}}(\mathbb{Z})$ and $Y:=\left(y_{n}\right) \in \ell_{\Phi_{2}}(\mathbb{Z})$ then $X Y \in \ell_{\Phi_{3}}(\mathbb{Z})$ with

$$
\|X Y\|_{\ell_{\Phi_{3}}(\mathbb{Z})} \leq 2\|X\|_{\ell_{\Phi_{1}}(\mathbb{Z})}\|Y\|_{\ell_{\Phi_{2}}(\mathbb{Z})}
$$

Proof. Let $t_{1}, t_{2} \geq 0$. Without loss of generality, suppose that $\Phi_{1}\left(t_{1}\right) \leq \Phi_{2}\left(t_{2}\right)$. By Lemma 1, we have

Hence

$$
t_{1} t_{2} \leq \Phi_{1}^{-1}\left(\Phi_{1}\left(t_{1}\right)\right) \Phi_{2}^{-1}\left(\Phi_{2}\left(t_{2}\right)\right) \leq \Phi_{1}^{-1}\left(\Phi_{2}\left(t_{2}\right)\right) \Phi_{2}^{-1}\left(\Phi_{2}\left(t_{2}\right)\right) \leq \Phi_{3}^{-1}\left(\Phi_{2}\left(t_{2}\right)\right)
$$

$$
\Phi_{3}\left(t_{1} t_{2}\right) \leq \Phi_{3}\left(\Phi_{3}^{-1}\left(\Phi_{2}\left(t_{2}\right)\right)\right) \leq \Phi_{2}\left(t_{2}\right) \leq \Phi_{1}\left(t_{1}\right)+\Phi_{2}\left(t_{2}\right)
$$

Since, $\Phi$ is convex function, we have

$$
\sum_{k=1}^{\infty} \Phi_{3}\left(\frac{\left|x_{k} y_{k}\right|}{2\|X\|_{\ell_{\Phi_{1}}(\mathbb{Z})}\|Y\|_{\ell_{\Phi_{2}}(\mathbb{Z})}}\right) \leq \frac{1}{2} \sum_{k=1}^{\infty} \Phi_{3}\left(\frac{\left|x_{k} \| y_{k}\right|}{\|X\|_{\ell_{\Phi_{1}}(\mathbb{Z})}\|Y\|_{\ell_{\Phi_{2}}(\mathbb{Z})}}\right)
$$


Furthermore, using Lemma 2 we get

$$
\sum_{k=1}^{\infty} \Phi_{3}\left(\frac{\left|x_{k}\right|\left|y_{k}\right|}{\|X\|_{\ell_{\Phi_{1}}(\mathbb{Z})}\|Y\|_{\ell_{\Phi_{2}}(\mathbb{Z})}}\right) \leq \sum_{k=1}^{\infty} \Phi_{1}\left(\frac{\left|x_{k}\right|}{\|X\|_{\ell_{\Phi_{1}}(\mathbb{Z})}}\right)+\sum_{k=1}^{\infty} \Phi_{2}\left(\frac{\left|y_{k}\right|}{\|Y\|_{\ell_{\Phi_{1}}(\mathbb{Z})}}\right) \leq 2 .
$$

Whenever $X:=\left(x_{n}\right) \in \ell_{\Phi_{1}}(\mathbb{Z})$ and $Y:=\left(y_{n}\right) \in \ell_{\Phi_{2}}(\mathbb{Z})$. Based on definition of $\|\cdot\|_{\ell_{\Phi_{3}}(\mathbb{Z})}$ we have $\|X Y\|_{\ell_{\Phi_{3}}(\mathbb{Z})} \leq 2\|X\|_{\ell_{\Phi_{1}}(\mathbb{Z})}\|Y\|_{\ell_{\Phi_{2}}(\mathbb{Z})}$, as desired.

Now, we present the sufficient condition for generalized Hölder's inequality in sequence Orlicz spaces.

Theorem 4. For $m \geq 2$. Let $\Phi_{i}, \Phi$ be Young functions for $i=1,2,3, \ldots, m$. If

$$
\prod_{i=1}^{m} \Phi_{i}^{-1}(\mathrm{t}) \leq \Phi^{-1}(\mathrm{t})
$$

for $t>0$, then for every $X_{i} \in \ell_{\Phi_{i}}(\mathbb{Z}), i=1,2,3, \ldots, m-1$ we have $\prod_{i=1}^{m} X_{i} \in \ell_{\Phi}(\mathbb{Z})$ with

$$
\left\|\prod_{i=1}^{m} X_{i}\right\|_{\ell_{\Phi}(\mathbb{Z})} \leq \mathrm{m} \prod_{i=1}^{m}\left\|X_{i}\right\|_{\ell_{i}(\mathbb{Z})} .
$$

Proof. . Let $\prod_{i=1}^{m} \Phi_{i}^{-1}(\mathrm{t}) \leq \Phi^{-1}(\mathrm{t})$ holds for $t>0$. By Lemma 1, we have

$$
t_{i} \leq \Phi_{i}^{-1}\left(\Phi_{i}\left(t_{i}\right)\right) \leq \Phi_{i}^{-1}\left(\sum_{i=1}^{m} \Phi_{\mathrm{i}}\left(t_{i}\right)\right)
$$

for $i=1,2,3, \ldots, m$. Hence we have

$$
\prod_{i=1}^{m} t_{i} \leq \prod_{i=1}^{m} \Phi_{i}^{-1}\left(\sum_{i=1}^{m} \Phi_{\mathrm{i}}\left(t_{i}\right)\right) \leq \Phi^{-1}\left(\sum_{i=1}^{m} \Phi_{\mathrm{i}}\left(t_{i}\right)\right)
$$

Since, $\Phi_{\mathrm{m}}$ is an increasing function, we have

$$
\Phi\left(\prod_{i=1}^{m} t_{i}\right) \leq \Phi\left(\Phi_{m}^{-1}\left(\sum_{i=1}^{m} \Phi_{\mathrm{i}}\left(t_{i}\right)\right)\right) \leq \sum_{i=1}^{m} \Phi_{\mathrm{i}}\left(t_{i}\right) .
$$

Now, let $X_{i} \in \ell_{\Phi_{i}}(\mathbb{Z}), i=1,2,3, \ldots, m$. Since $\Phi$ convex function and using Lemma 2, we have

$$
\sum_{k=1}^{\infty} \Phi\left(\frac{1}{m} \prod_{i=1}^{m} \frac{\left|x_{k}^{(i)}\right|}{\|X\|_{\ell_{\Phi_{i}}(\mathbb{Z})}}\right) \leq \frac{1}{m} \sum_{k=1}^{\infty} \Phi\left(\prod_{i=1}^{m} \frac{\left|x_{k}^{(i)}\right|}{\|X\|_{\ell_{\Phi_{i}}(\mathbb{Z})}}\right) \leq \frac{1}{m} \sum_{i=1}^{m} \sum_{k=1}^{\infty} \Phi\left(\frac{\left|x_{k}^{(i)}\right|}{\|X\|_{\ell_{\Phi_{i}}(\mathbb{Z})}}\right) \leq 1 .
$$

Whenever $X_{i}:=\left(x_{k}^{(i)}\right) \in \ell_{\Phi_{i}}(\mathbb{Z})$. By using the definition of $\|\cdot\|_{\ell_{\Phi}(\mathbb{Z})}$ we have

as desired.

$$
\left\|\prod_{i=1}^{m} X_{i}\right\|_{\ell_{\Phi}(\mathbb{Z})} \leq \mathrm{m} \prod_{i=1}^{m}\left\|X_{i}\right\|_{\ell_{i}(\mathbb{Z})}
$$




\section{Conclusion}

In this short paper, We have shown the sufficient condition for generalized Holder's inequality in of sequence Orlicz spaces. From Theorem 2.1 in [10], we can state that the condition $\prod_{i=1}^{m} \Phi_{i}^{-1}(\mathrm{t}) \leq \Phi^{-1}(\mathrm{t})$ for $t>0$ is sufficient condition for generalized Holder's inequality in continuous Orlicz spaces and Orlicz sequence spaces.

Acknowledgment. This research was made possible by the following grants supports: Hibah Penguatan Kompetensi UPI and Hibah Afirmasi dan Pembinaan Dosen UPI 2019.

\section{References}

[1] Orlicz, W.: Linear Functional Analysis (Series in Real Analysis Volume 4). World Scientific, Singapore. (1992)

[2] Kufner, A., John, O., and Fuc ïk, S.: Function Spaces. Noordhoff International Publishing, Czechoslovakia (1977).

[3] Rao, M. M. and Ren Z. D.: Theory of Orlicz spaces. Volume 146 of Monographs and Textbooks in Pure and Applied Mathematics. Marcel Dekker Inc., New York (1991)

[4] Maligranda, L. and Mastylo: M. Inclusion Mappings between Orlicz Sequence Spaces. Journal of Functional Analysis Vol. 176, pp. 264-279 (2000)

[5] Masta, A. A., Gunawan, H., and Budhi, W. S.: Inclusion property of Orlicz and weak Orlicz spaces. J. Math. Fund. Sci. 48-3. pp. 193-203 (2016)

[6] Masta, A. A., Ifronika, and Fatimah, S.: Sufficient and Necessary Conditions for Holder's Inequality in Weighted Orlicz Spaces, to appear in International Journal of Applied Physics and Mathematics 2019

[7] Osançlıol, A.: A Note On The Definition of An Orlicz Space. Afyon Kocatepe University Journal of Science and Engineering, pp. 1-6 (2015)

[8] Luxemburg, W. A. J.: Banach Function Spaces. Thesis. Technische Hogeschool te Delft. 1955

[9] Masta, A.A.: On Uniform Orlicz Spaces. Thesis. Bandung Institute of Technology (2013)

[10] Ifronika, Masta Al A, Nur, M, and Gunawan H 2019 Generalized Hölder's inequality in Orlicz spaces 